\title{
Differential gene expression in nearly isogenic lines with QTL for partial resistance to Puccinia hordei in barley
}

Xinwei Chen ${ }^{1 *}$, Rients E Niks ${ }^{2}$, Peter E Hedley ${ }^{1}$, Jenny Morris ${ }^{1}$, Arnis Druka ${ }^{1}$, Thierry C Marcel ${ }^{2,3}$, Anton Vels ${ }^{2}$, Robbie Waugh ${ }^{1 *}$

\begin{abstract}
Background: The barley-Puccinia hordei (barley leaf rust) pathosystem is a model for investigating partial disease resistance in crop plants and genetic mapping of phenotypic resistance has identified several quantitative trait loci (QTL) for partial resistance. Reciprocal QTL-specific near-isogenic lines (QTL-NILs) have been developed that combine two QTL, Rphq2 and Rphq3, the largest effects detected in a recombinant-inbred-line (RIL) population derived from a cross between the super-susceptible line L94 and partially-resistant line Vada. The molecular mechanism underpinning partial resistance in these QTL-NILs is unknown.
\end{abstract}

Results: An Agilent custom microarray consisting of 15,000 probes derived from barley consensus EST sequences was used to investigate genome-wide and QTL-specific differential expression of genes 18 hours post-inoculation (hpi) with Puccinia hordei. A total of 1,410 genes were identified as being significantly differentially expressed across the genome, of which 55 were accounted for by the genetic differences defined by QTL-NILs at Rphq2 and Rphq3. These genes were predominantly located at the QTL regions and are, therefore, positional candidates. One gene, encoding the transcriptional repressor Ethylene-Responsive Element Binding Factor 4 (HvERF4) was located outside the QTL at $71 \mathrm{cM}$ on chromosome $1 \mathrm{H}$, within a previously detected eQTL hotspot for defence response. The results indicate that Rphq2 or Rphq3 contains a trans-eQTL that modulates expression of HvERF4. We speculate that HvERF4 functions as an intermediate that conveys the response signal from a gene(s) contained within Rphq2 or Rphq3 to a host of down-stream defense responsive genes. Our results also reveal that barley lines with extreme or intermediate partial resistance phenotypes exhibit a profound similarity in their spectrum of Ph-responsive genes and that hormone-related signalling pathways are actively involved in response to Puccinia hordei.

Conclusions: Differential gene expression between QTL-NILs identifies genes predominantly located within the target region(s) providing both transcriptional and positional candidate genes for the QTL. Genetically mapping the differentially expressed genes relative to the QTL has the potential to discover trans-eQTL mediated regulatory relays initiated from genes within the QTL regions.

\section{Background}

Plants have evolved complex mechanisms to defend against pathogen attack. Two types of immunity have been described: Pathogen-Associated Molecular Pattern (PAMP)-Triggered Immunity (PTI) and Effector-Triggered Immunity (ETI). PTI is induced at an early stage when PAMPs are recognized by Pattern Recognition Receptors

\footnotetext{
*Correspondence: xinwei.chen@scri.ac.uk; robbie.waugh@scriac.uk ${ }^{1}$ Genetics Programme, Scottish Crop Research Institute, Dundee, UK Full list of author information is available at the end of the article
}

(PRRs), whereas ETI is induced by direct or indirect association of a Resistance $(\mathrm{R})$ protein with a pathogen-derived effector [1-4]. The outcomes of the two immune systems appear to be partial or quantitative resistance and non-host resistance (PTI), and qualitative resistance (ETI). Recently, Niks and Marcel [5] proposed that the varying efficacy of PTI suppression by pathogen effectors may explain partial resistance. In cereal crops, the barley-Puccinia hordei Otth (barley leaf rust) pathosystem is a model for investigating partial and non-host resistance. Microscopic studies on resistance levels in relation to the pathogen developmental
C Biomed Central

C 2010 Chen et al; licensee BioMed Central Ltd. This is an Open Access article distributed under the terms of the Creative Commons Attribution License (http://creativecommons.org/licenses/by/2.0), which permits unrestricted use, distribution, and reproduction in any medium, provided the original work is properly cited. 
phases has indicated plant cell wall penetration and haustorium formation by $P$. hordei as critical phases determining the success or failure of the infection [6]. Pre-haustorial resistance reduces the chance of successful haustorium formation by the fungal pathogen in the host cells. Failed attempts are typically associated with cell wall appositions [6-10]. Such pre-haustorial basal host defence is a typical reaction to $P h$-infection in most (if not all) barley lines exhibiting partial resistance [6]. Post-haustorial resistance is usually due to $R$ gene-mediated hypersensitive response after haustorium formation [9].

These two types of resistance have strategic significance in plant breeding for resistance to diseases. Quantitative or partial resistance has become increasingly important because of its broader spectrum and higher durability compared to $R$-gene mediated race-specific resistance. Many of the genes underlying partial resistance have plant developmental stage-dependent effectiveness [11]. Currently, over 20 quantitative trait loci (QTL) for quantitative basal resistance to leaf rust from five different mapping populations have been mapped to barley chromosomes [11-16]. They are named $R p h q$ genes [Resistance to Puccinia hordei (quantitative)]. Of these, 10 were effective during the seedling stage, and were detected by QTL analysis of the latency period exhibited by the rust fungus on seedling leaves [15]. Considerable effort has been expended in an attempt to identify the genes underlying these QTL. Notably, a set of NILs and reciprocal NILs have been developed that contain single $(R p h q 2,3,4)$ or combined $(R p h q 2+3)$ introgressed segment(s) carrying resistance and susceptibility QTL allele(s) that were identified in an L94 $\times$ Vada RIL population $[11,16,17]$. L94 is an Ethiopian landrace and highly susceptible to barley leaf rust. Vada is a Dutch cultivar expressing a high level of partial resistance. Following a positional cloning strategy, Marcel et al. [18] have fine-mapped Rphq2, the QTL with largest effect, to an interval of $0.11 \mathrm{cM}$ corresponding to less than $200 \mathrm{~kb}$ in physical length.

Microarray technology is being widely used to address various biological, biochemical and genetic questions. Microarray-based gene expression studies can be generally grouped into two major categories. The first aims to address specific biological questions by monitoring the differential expression of genes under contrasting conditions or over time. The most common studies in this field are the investigations on host-pathogen interactions. Profiling changes in genome-wide expression in response to pathogen challenge has identified a large spectrum of genes that are responsive to pathogen attack or are associated with plant resistance in various pathosystems (reviewed by Wise et al. [19]). The second category is based on the more recently emerged concept of 'genetical genomics' [20] or expression QTL (eQTL) mapping that combines highly parallel gene expression studies with the power of genetic segregation. eQTL studies have been performed on maize, eucalyptus and Arabidopsis [21]. eQTL analyses in barley have addressed the global genetic architecture of transcript abundance in

[22], the phenomenon of limited pleiotropy [23] and as an approach to identify the causal or candidate genes underlying partial resistance to fungal diseases [24,25]. While both categories of microarray studies are based on variation in transcript abundance, eQTL analysis provides a genetic dimension that can differentiate cisfrom trans-regulation and the genetic locations of a large number of genes through the co-location of high LOD eQTL (i.e. highly differentially expressed) and their structural genes [26]. This is particularly valuable for a crop with large and unsequenced genome like barley.

Here, using a previously reported Agilent $15 \mathrm{k}$ custom array [25], we performed differential expression analysis of QTL-NILs and their recurrent parental lines at 18 hours post-inoculation (hpi) with Puccinia hordei. Our major objective was to identify candidate genes for $R p h q 2$ and $R p h q 3$. In addition, transcript profiles between $P h$-infected parents and their respective mockinoculated controls allowed the establishment of transcriptomic signatures for each line in response to $P h$-infection. Our results indicate that transcriptional differentiation between QTL-NILs and their respective recurrent parents reveals components of a regulatory transcriptional relay induced in response to $\mathrm{Ph}$-infection. The datasets generated offer a basis for further studies on defence signalling in relation to partial resistance to $P$. hordei in barley.

\section{Results}

Transcriptomic signatures of response to $P$. hordei $(\mathrm{Ph}$ infected vs. Mock inoculated)

Plant defence responses involve transcriptional activation of a plethora of specific genes and regulation of their temporal and spatial expression [27]. To investigate the genome-wide transcriptional signatures of susceptible and partially resistant barley lines L94 and Vada respectively in response to $P$. hordei infection, we compared $P h$-infected with mock-inoculated leaf material. A stringent threshold with fold change $>2$ and false discovery rate $($ FDR $)<0.05$ was adopted for declaring significant differences. At this threshold, 669 and 514 genes were respectively identified in L94 and Vada as 'significantly differentially expressed' with 381 (362 up + 19 down) overlapping between the two lines while 421 (L94 175 up +113 down and Vada 121 up +12 down) were present in only one of the two parents (Figure 1 ). This yielded a total of 802 genes which we considered 'Ph-responsive'. Close examination of the expression data of the 421 


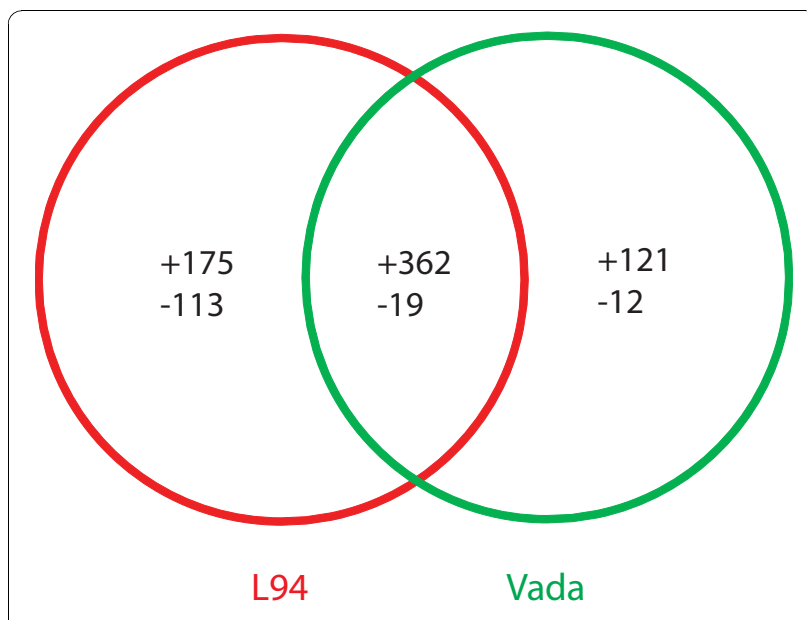

Figure 1 Venn diagram showing number of $P h$-responsive genes (fold change $>2$, FDR $<0.05$ ) identified in L94 and Vada. ' + ' and '-' represent up- and down-regulation respectively.

'Ph-responsive' genes from both parents showed that while a substantial number failed to meet the stringent thresholds applied (fold change $>2$, FDR $<0.05$ ) they still exhibited statistically significant differential expression in both parents. Therefore, a relaxed threshold ignoring the fold changes was adopted for the follow-up analysis on the commonality and specificity of response to $\mathrm{Ph}$ infection between the resistant and susceptible lines using all $802 \mathrm{Ph}$-responsive genes. We plotted the logtransformed expression ratios of Vada against L94 and classified them into four groups. Genes that showed the same expression patterns (up- or down-regulation) and expression changes at $p<0.05$ in both lines were defined as being common to both lines (Figure 2, black empty circles), whereas, those that showed significant expression changes in one line but no significant expression changes $(p>0.5)$ or a contrasting expression pattern in the other line were considered as being line-specific (Figure 2, red empty circles for Vada and blue for L94). The remaining genes that had no strong evidence to suggest either commonality or specificity were grouped into 'not determined' (Figure 2, green empty circles). There were a total of 584, 24, 34 and 160 genes that appeared to be in common, Vada- or L94-specific or 'not determined' representing $73 \%, 3 \%, 4 \%$ and $20 \%$ of the $802 \mathrm{Ph}$-responsive genes respectively. Figure 3 shows a colour-coded heat map that was converted from the relative expression ratios (signal intensity from $\mathrm{Ph}$-infected $v s$. mockinoculated controls) of the 802 genes and the $58(24+$ 34) line-specific genes showing the overall similarity and specificity of gene expression in L94 and Vada. Full expression information of the 802 genes and the line-specific genes is given in the Additional File 1 (Table S1) and 2 (Table S2) respectively.
Genome-wide $P h$-responsive genes have previously been investigated [25] using Steptoe (St) and Morex $(\mathrm{Mx})$, two barley cultivars with similar, intermediate levels of resistance to $P$. hordei (leaf materials were prepared from the same experiment as the current study with L94 and Vada). We therefore compared the data from L94/Vada with those from St/Mx. At exactly the same thresholds (i.e. FC $>2$, FDR $<0.05$ ) a total of 1154 genes were identified as $\mathrm{Ph}$-responsive in St/Mx [25]. Applying exactly the same criteria as described above, we identified 913 (79\%), 21 (1.8\%), and 19 (1.6\%) genes that were common, St-specific and Mx-specific respectively. We then explored the common genes in each of these categories between the two experiments (Table 1). $75.4 \%$ (605) of the 802 genes detected with L94/Vada were also detected with $\mathrm{St} / \mathrm{Mx}$ and more than half of the genes (466) were significant in all four lines, highlighting the similarity of response to $P h$-infection across genotypes. Of the 24 and 34 genes that were specifically detected in Vada and L94 respectively, 13 and six of these were reproducibly identified as $P h$-responsive in Steptoe or Morex (Additional File 3, Table S3). All of the 13 Vada (resistant)-specific genes were up-regulated in Vada and St or Mx. Ten of these genes showed significant differential expression $(p<0.05)$ between St and Mx. Of the six L94 (susceptible)-specific genes, only one up-regulated gene (unigene21775) showed significant differential expression between St and Mx (Additional File 3, Table S3).

To further characterise the biological processes represented by the $802 \mathrm{Ph}$-responsive genes, we performed gene ontology (GO) analysis by classifying the $P h$ responsive genes into functional biological categories based on GO terms retrieved from their rice homologues through the rice database at http://rice.plantbiology. msu.edu/annotation_pseudo_goslim.shtml. The Phresponsivegenes were associated with a broad range of biological processes. The primary category was related to defence response. We further classified these genes into 11 major functional categories following the GO terms in 'biological process' with all remaining genes grouped into 'other functions' or 'unknown'. The results are shown in Figure 4. They indicate that at the sampling time point of $18 \mathrm{hpi}$, the plants had responded to defend against the $P h$-infection.

\section{Differential expression analysis between Ph-infected recurrent parents (L94 vs. Vada)}

We performed genome-wide differential expression analysis by comparing expression differences between $P h$ infected L94 and Ph-infected Vada at 18 hpi. A total of 1411 genes were identified as being differentially expressed (FC >2, FDR <0.05), of which 247 were $P h$ responsive genes as described above. The majority 


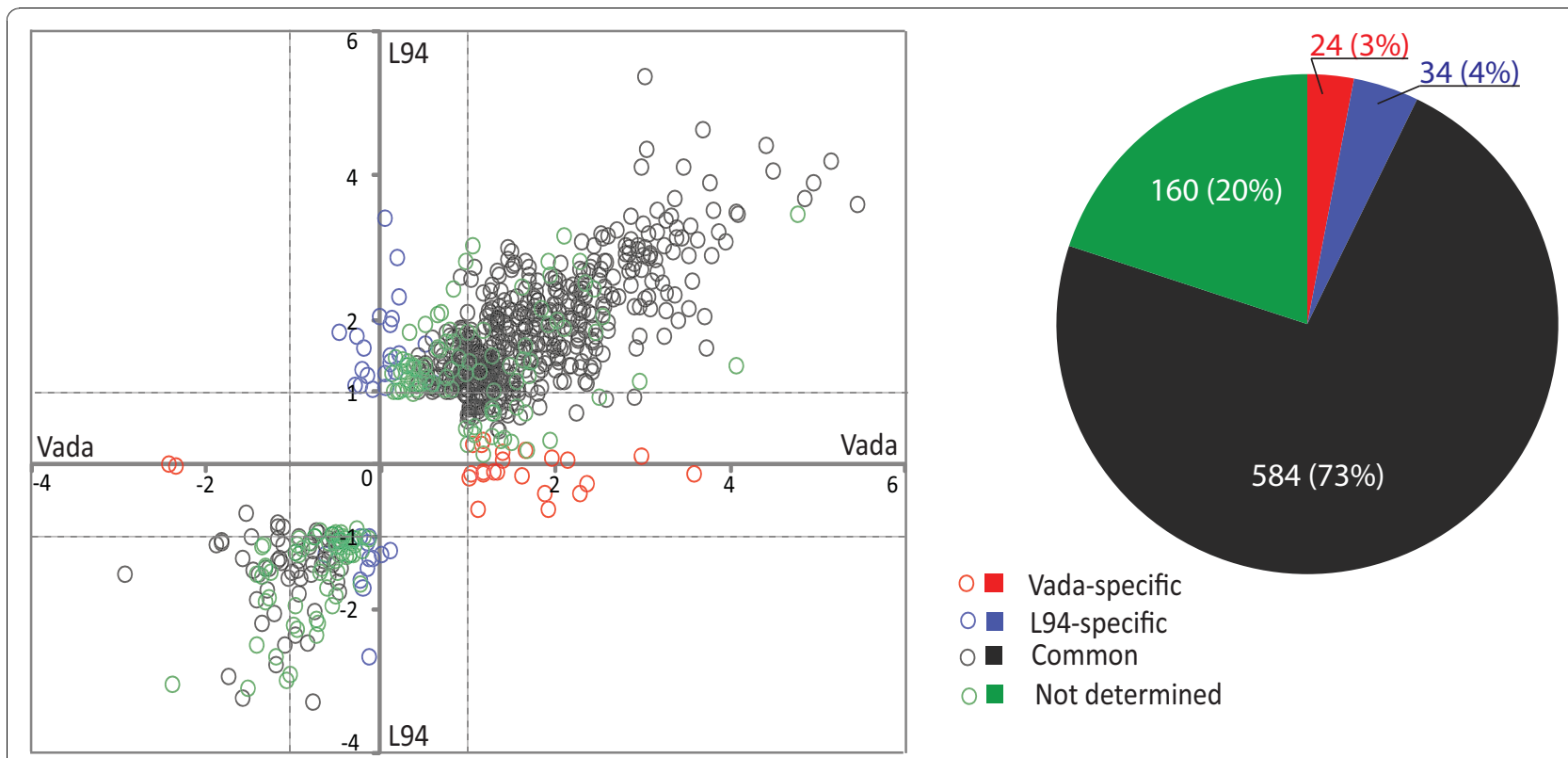

Figure 2 Scatter plot of log ratios (ratio of signal intensity Ph-infected/Mock control) of the 802 Ph-responsive genes from Vada (horizontal axis) and L94 (vertical axis). Colour-coded circles represent genes in different groups with proportions shown in the pie chart. Log ratios $>0$ or $<0$ indicates up- or down-regulation respectively, dashed lines set at 1 and -1 corresponding to $2 x$ fold change in expression.

(1164) represent genome-wide, genotype-specific differences in gene expression. The detailed information of these genes regarding their expression ratios, $p$-values and functional annotations is presented in Additional File 4 (Table S4).

\section{Differential expression between Ph-infected QTL-NILs and Ph-infected recurrent parents}

To identify QTL-specific and differentially expressed genes accounted for by genetic differences in the QTL regions, the two reciprocal QTL-NILs were compared with their respective recurrent parents: L94 vs. L94$R p h q 2+3$ and Vada $v s$. Vada-Rphq2+3. A total of 94 genes were identified as significant $(\mathrm{FC}>2$, FDR $<0.05)$ in at least one comparison. Of these, 39 genes showed a significant difference in one recurrent parent/QTL-NIL comparison but not with the other. We attribute these observations to the different size and incomplete overlap of the introgressed segments in the two recurrent parent/QTL-NIL pairs. These genes were, therefore, not pursued further. The remaining 55 genes showed expression differences at $p<0.05$ in both comparisons and were, therefore, considered potentially relevant to the QTL regions. This suggests that differential expression results from genetic factors differing specifically within the QTL regions (Additional File 5, Table S5, and Figure 5). Of these 55 genes, 50 were present on the list of 1411 differentially expressed genes between $P h$-infected Vada and $P h$-infected L94. The remaining five genes (Table S5, underlined) did not fulfil the criteria $(\mathrm{FC}>2$, FDR $<0.05)$ set for the differential expression between the two $\mathrm{Ph}$-infected recurrent parents, but their expression differences were still statistically significant $(p<0.05)$.

\section{Transcription of QTL-specific and differentially expressed} genes in response to $P h$-infection

To identify whether the 55 QTL-specific and differentially expressed genes were also $P h$-responsive, expression data from the $P h$-infected $v s$. Mock-inoculated experiment was re-investigated (Table S5). Six genes showed changes that fulfilled the criteria (fold change $>2$, FDR $<0.05$ ) set for defining $P h$-responsive genes. Twelve genes did not fully meet the criteria, but their level of differential expression was still statistically significant $(p<0.05)$ in at least one of the lines. The others were not statistically significant.

\section{Identification of positional candidates for Rphq2 and Rphq3}

To determine the map position of the 55 QTL-specific and differentially expressed genes, we took advantage of available datasets previously generated in three different eQTL studies (germinating embryos [22]; P. tritici infected leaves http://genenetwork.org, R. Wise, unpublished data) and $P h$-infected seedling leaves [25]. 52 of the 55 genes had one or more eQTL in at least one of these three experiments, yielding 163 eQTL in total. The distribution of these eQTL was investigated by plotting their map positions against their LOD/LRS values 


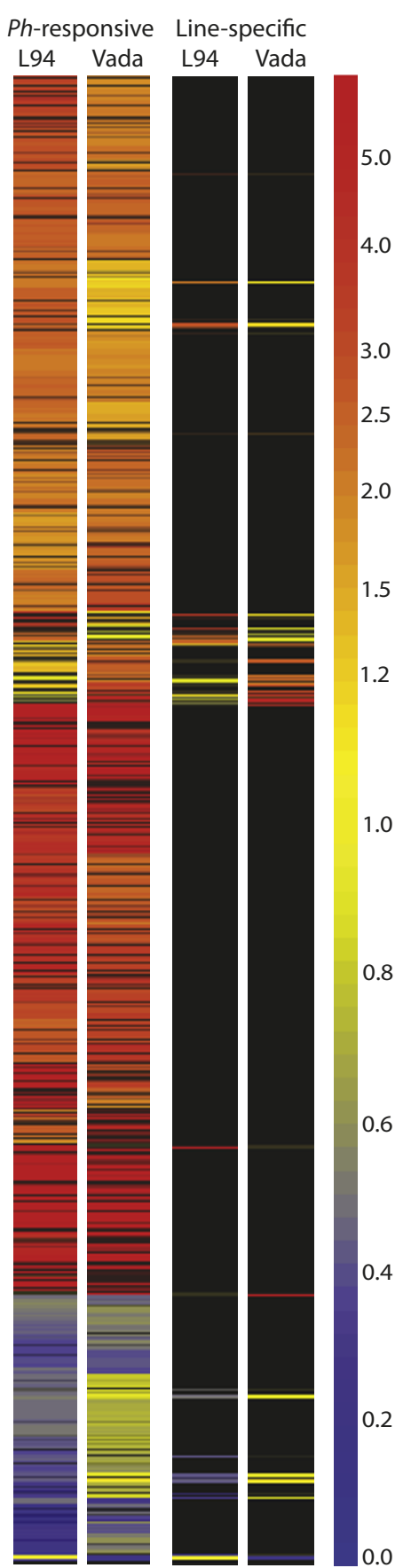

Figure $3 \mathrm{~A}$ heat map illustrating expression patterns of the $802 \mathrm{Ph}$-responsive genes identified in L94 and Vada. Genes are organized by 'gene tree' hierarchical clustering implemented in GeneSpring based on overall similarity in expression patters (the gene tree has been omitted for clarity). The color bar indicates the expression ratios of the two treatments (Ph-infection vs. mockinoculated controls). Red and blue represent up- and downregulation respectively, whereas yellow represent no significant alteration. Left panel shows 802 genes that were significantly (FC $>2$, FDR <0.05) altered in at least one of the two lines; right panel shows the 58 line-specific genes that were only significantly ( $F C>2$, FDR $<0.05)$ altered in one line but not the other.
(Figure 6). 40 genes with eQTL mapped to within the QTL regions (nine at $R p h q 2$ and 31 at $R p h q 3$ ) (Figure 6, Table S5), of which 33 (83\%) had LOD >10 or LRS $>50$ suggesting they are cis-eQTL (i.e. their structural genes map to the same locus as the eQTL). We then explored three available gene-based mapping datasets: Illumina OPA-SNPs [28], Single Feature Polymorphisms (R. Wise, unpublished data) and TDMs [22] to help assign genetic map positions to the 55 genes. This allowed four and nine genes to be placed within the confidence intervals of $R p h q 2$ and $R p h q 3$ respectively. All of these genes overlapped with the eQTL except two (unigene7920 and 2826) for which no eQTL was detected in the three eQTL studies (Table S5). Rphq2 and $R p h q 3$ on chromosome $2 \mathrm{H}$ and $6 \mathrm{H}$ are syntenic to regions on rice chromosomes Os04 and Os02 respectively. Conservation of synteny allowed us to infer the approximate map positions of an additional 15 genes to within the QTL regions (Table S5). Thus, of the 55 QTL-specific differentially expressed genes, the map location of nine and 34 fell within Rphq2 and Rphq3 respectively, whilst the 11 others remain unknown. Of note was the observation that one gene (unigene6636), encoding an Ethylene-Responsive Transcription Factor 4 (HvERF4) (rice orthologue Os05g41780.1), has been mapped as Illumina OPA-SNP marker 11_10686 to chromosome $1 \mathrm{H}$ at position $71 \mathrm{cM}$ [28]. This map position is consistent with a location based on conservation of synteny between rice Os05 and barley chromosome $1 \mathrm{H}$, suggesting that differential expression of this gene is the consequence of trans-regulation by a gene located within either $R p h q 2$ or $R p h q 3$.

\section{Discussion}

In this study, we performed differential expression analysis of two reciprocal QTL-NILs and compared them with their respective recurrent parents. As QTL-NILs differ genetically from their recurrent parent only in the selected QTL regions, we would anticipate that genetic polymorphism between these QTL regions would account for any differential expression observed. However, due to the complexity of gene regulation, differentially expressed genes may not necessarily be located in the introgressed QTL regions, which may themselves contain regulatory genes affecting the expression of other genes spread throughout the genome. We therefore established the map positions of differentially expressed genes by exploiting previously generated gene mapping datasets. Of the 55 genes highlighted in our comparisons between NILs and recurrent parents, 40 detected eQTL in at least one of the three previous eQTL studies and co-located at the QTL regions, most (83\%) having high LOD/LRS scores [22,25] (Table S5). eQTL with high LOD scores have been demonstrated 
Table 1 Number of overlapping genes (shown in matrix) in different categories detected in two experiments with St/ Mx and Vada/L94

\begin{tabular}{lcccc}
\hline & Ph-responsive (1154) & Common (913) & St-specific (21) & Mx-specific (19) \\
\hline Ph-responsive (802) & 605 & 532 & 9 & 5 \\
Common (584) & 506 & 466 & 5 & 3 \\
Vada-specific (24) & 13 & 6 & 3 & 2 \\
L94-specific (34) & 6 & 3 & 0 & 1 \\
\hline
\end{tabular}

Note: $P$-responsive genes were selected on criteria with fold change $>2$ and FDR $<0.05$; genes with similar patterns were selected from $P h$-responsive gene on $p<0.05$ without considering fold changes.

previously to be almost always cis-eQTL [22,25,26] placing these genes within the Rphq2 or Rphq3 QTL regions. The observation that so many significantly differentially expressed genes appeared to be regulated in cis- is in agreement with previous studies [25,29]. An exception was unigene 6636, encoding HvERF4. This gene mapped to $71 \mathrm{cM}$ on chromosome $1 \mathrm{H}$, consistent with the position of its rice homologue Os05g41780.1 predicted by conservation of synteny [28]. This observation raises the possibility that the introgressed regions at either Rphq2 or Rphq3 contain a polymorphic transacting regulator that differentially modulates expression of $H v E R F 4$. No eQTL for HvERF4 was detected at the regions corresponding to Rphq2 or Rphq3 in the St/Mx $\mathrm{DH}$ mapping population, consistent with the fact that it does not segregate for Rphq2 or Rphq3.

HvERF4 is a member of a family of plant transcription factors functionally involved in defence signalling pathways related to ethylene, jasmonic acid and abscisic acid. Over-expression of Arabidopsis AtERF4 represses the expression of pathogenesis-related (PR) genes such as basic chitinase and beta-1,3-glucanase genes and genes containing a GCC-box [30], the core sequence element of promoters required for responsiveness to ethylene [31]. In our previous experiment with Steptoe and Morex, cultivars with similar but intermediate levels of partial resistance to leaf rust, we also observed that $H v E R F 4$ was significantly up-regulated by $P h$-infection but no differential expression $(p>0.2)$ was observed between the parents [25]. Here, HvERF4 was induced in Ph-infected L94 (susceptible) $(\mathrm{FC}=4.42)$ and Vada (partially resistant) $(\mathrm{FC}=2.42$ ) as compared to mockinoculated controls (Table S5), and the expression level of the Vada allele was only a third $(\mathrm{FC}=0.34)$ of that of the L94 allele after induction. The association of resistance/susceptibility with lower/higher expression of $H v E R F 4$ appears to be in agreement with the negative regulatory role of HvERF4 on the expression of PR and other defence responsive genes. However, consistent association of higher expression of PR genes with resistance was not observed in Vada and L94. This may reflect the general complexity of natural resistance response coupled with allelic variation at PR genes between these two lines. While this train of inference highlights HvERF4 as potentially important in this specific defense interaction, none of the so far reported 20 QTL for partial resistance to leaf rust, nor any of the QTL for resistance to heterologous rusts is co-located with HvERF4 at $71 \mathrm{cM}$ on chromosome $1 \mathrm{H}$ [11-16]. Thus, HvERF4 is not a positional candidate for any of

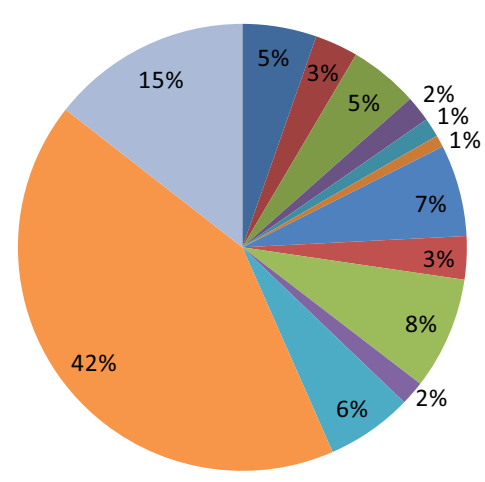

\begin{tabular}{rcl}
\hline+ & - & Function categories \\
\hline 36 & 7 & Transcription factors \\
25 & 0 & PR proteins \\
40 & 0 & Receptor and R gene (like) proteins \\
12 & 3 & Hormone related processes \\
11 & 0 & Phenylpropanoid pathway \\
6 & 1 & Phenylalanine and tryptophan metabolism \\
44 & 9 & Response to stress and stimulus \\
23 & 2 & Oxydative burst \\
59 & 6 & Signal perception and transduction \\
14 & 0 & Lipid binding or metabolic process \\
47 & 3 & Transporters \\
254 & 84 & Other functions \\
87 & 29 & Unknown \\
658 & 144 & Total \\
\hline
\end{tabular}

Figure 4 Functional classification of the $\mathbf{8 0 2} \mathbf{P h}$-responsive genes. Number of up (+) or down (-) regulated genes are shown in the table (see Additional File 1, Table S1 for details). 


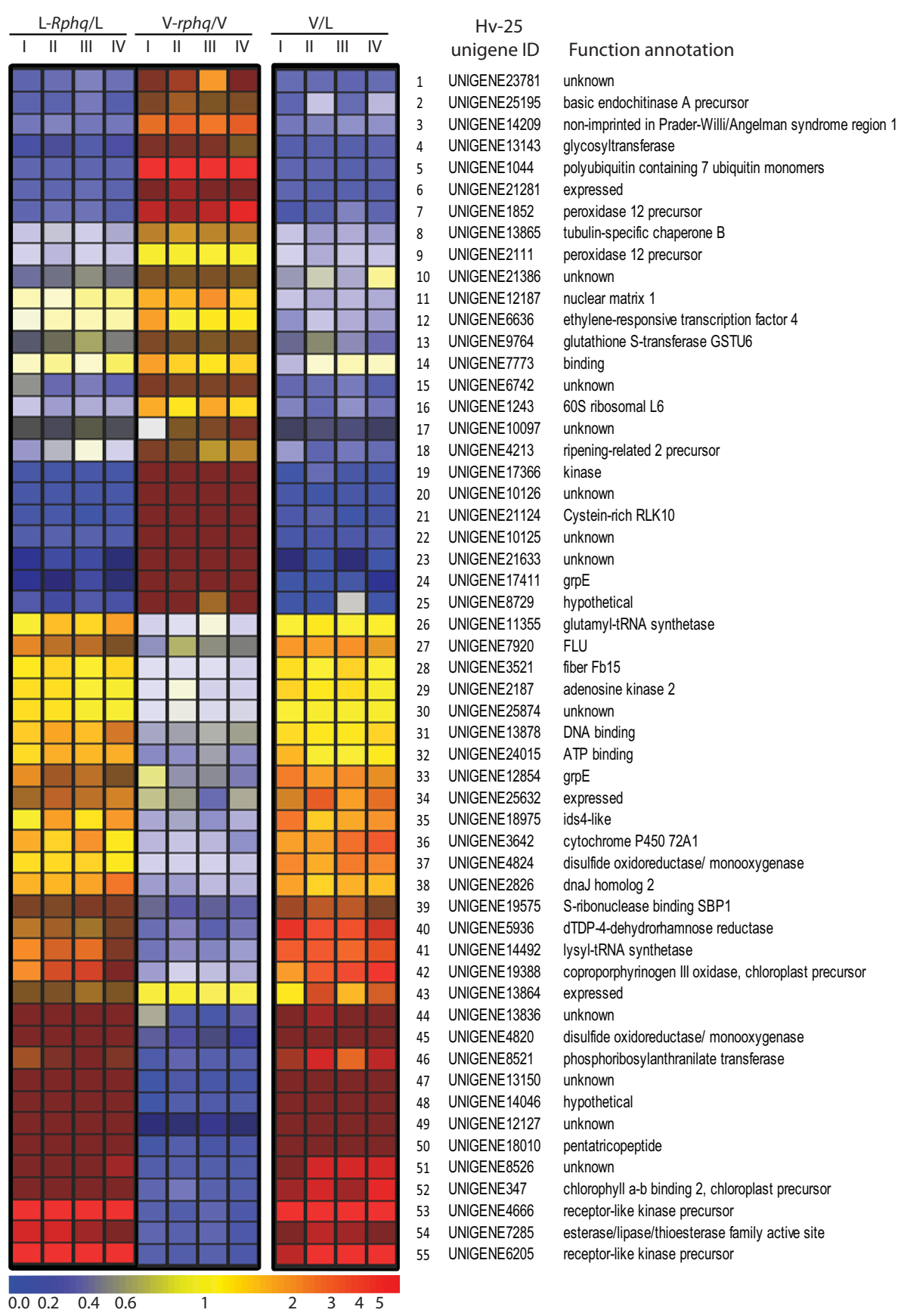

Figure 5 Heat map of the genes significantly and differentially expressed in the three comparisons. ' $L$ ' and ' $V$ ' on top of the heat map refer to L94 and Vada respectively. Roman numerals represent the four biological replicates. Colour coding represents the transcript abundance ratios. The two comparisons involving NILs were performed on microarray slide 3 and showed reversed colouring reflecting the reciprocal features of the NILs in their genetic background. Comparison between the two parents was conducted on microarray slide 2 with transcript abundance being calculated as L94/Nada. The genes (rows) and treatment groups (columns) are clustered through gene tree generation by GeneSpring program on distance (gene tree has been omitted for clarity).

the reported QTL. However, of direct relevance is a previously highlighted eQTL hotspot for

genes that was associated with OPA-SNP 11_20157 [25] at $70 \mathrm{cM}$ (98 cM on the consensus map [28]) on chromosome $1 \mathrm{H}$ spanning the region containing HvERF4. This hotspot comprised $127 \mathrm{eQTL}$ in less than a $10 \mathrm{cM}$ interval and contained genes primarily involved in defence response [25]. Given its known role in 


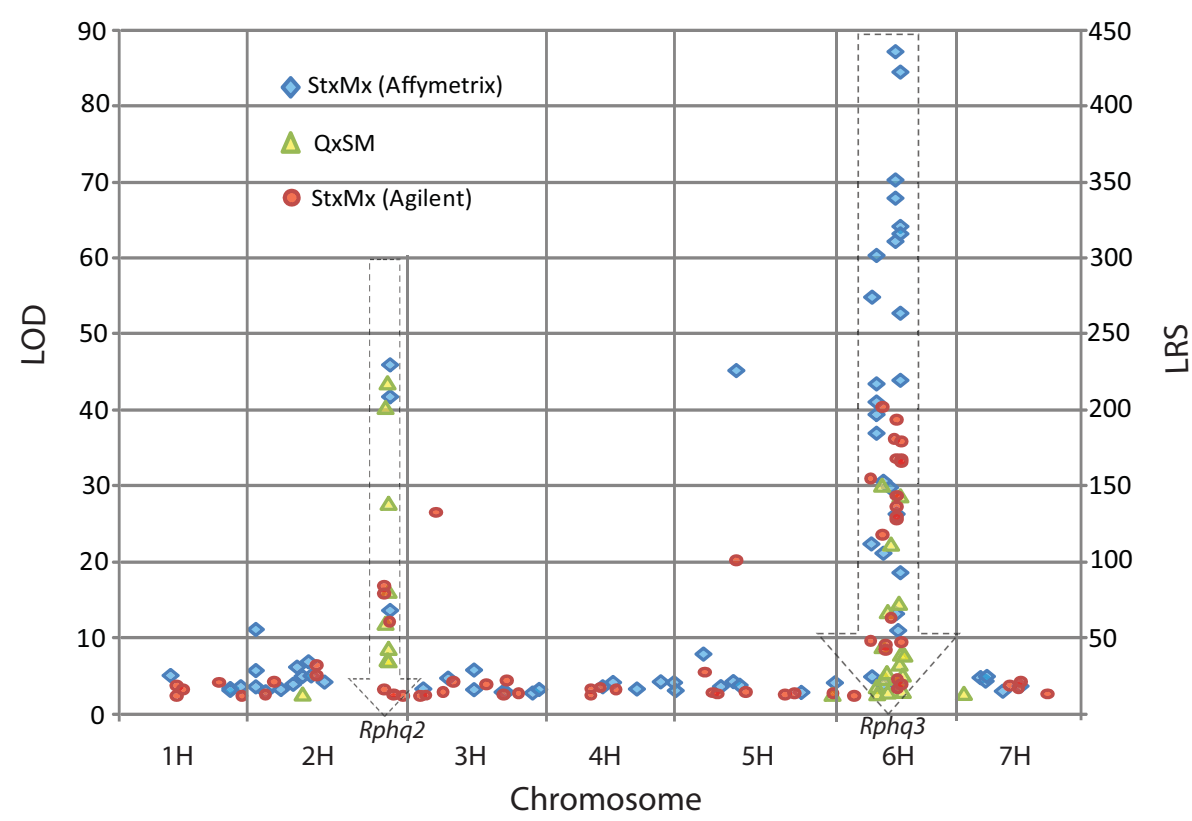

Figure 6 Distribution of the 163 eQTL detected from three experiments for the 52 genes (3 genes without eQTL detected) differentially expressed in QTL-specific NILs. Blue diamond, red dots and green triangles represent eQTL identified by Potokina et al. [22], Chen et al. [25] and Wise et al. (unpublished results) respectively. eQTL co-located with Rphq2 and Rphq3 were framed with dash-lined arrows. Significance levels of eQTL detected in the St/Mx population refer to LOD score, those with ' $Q \times S M^{\prime}$ ' population refer to LRS.

PR-protein regulation, we speculate that HvERF4 represents a key regulatory relay component of the signalling pathway that controls expression of at least a portion of the genes with eQTL located at the hotspot on chromosome $1 \mathrm{H}$. Considering these observations together we hypothesise that the causal genetic polymorphism at either $R p h q 2$ or $R p h q 3$ differentially regulates $H v E R F 4$ in trans (possibly through direct or indirect modulation of ethylene, jasmonic acid or abscisic acid levels, known in Arabidopsis to alter levels of AtERF4 expression [30]), the consequence of which is differential regulation of down-stream defence responses. In this scenario, the candidate genes for $R p h q 2$ or Rphq3 would be those acting up-stream rather than down-stream of HvERF4 and possibly involved directly or indirectly in plant hormone signalling pathways. While we did not find such a candidate from the annotated functions of the QTL-specific and differentially expressed candidates for $R p h q 2$ and $R p h q 3$, the gene controlling expression of HvERF4 may, however, not be differentially expressed between L94 and Vada, may not be on our expression platform (which probably contains less than half of the barley genes) or may not be at the orthologous position in rice. An alternative to identifying the causal gene for $R p h q 2$ or $R p h q 3$ could be through map-based cloning of the trans-eQTL for HvERF4.

Marcel et al. [18] narrowed down the genetic interval for $R p h q 2$ to $0.11 \mathrm{cM}$ corresponding to a physical length of $183 \mathrm{~kb}$ in barley (Marcel and Niks unpublished data) and a $69.7 \mathrm{~kb}$ syntenic region on rice chromosome 4 . Inspection of all predicted genes in the $R p h q 2$ syntenic interval in rice identified a cluster of six peroxidase genes and a MAP3K gene [18] as potential candidates because of their functional involvement in defence responses. In this study, we identified four barley genes at Rphq2 that were differentially expressed and had homologues located in the syntenic region in rice (Table S5, unigene 1852 (no.7), unigene2111 (no.9), unigene13865 (no. 8) and unigene8521 (no. 46)). Unigene1852 and 2111 both encode peroxidases and are within the $0.11 \mathrm{cM}$ interval containing Rphq2. The other two, according to the fine mapping data of Marcel et al. [18] fell just outside the candidate interval. However, given the frequent breakdown in conservation of synteny, positional candidate gene identification using this approach alone remains problematic. Differential expression in the QTL-NILs identified an additional five candidate genes (Figure 5 and Table S5: no. $2,3,19,21$ and 51 ) that were not apparently present in the syntenic region of rice. Two of these encode proteins that are functionally involved in signal transduction (Additional file 5, Table S5, no.19 and 21 encoding a kinase and a receptor-like kinase respectively), one PR protein, and one with homology to human NIPA1, implicated in Prader-Willi/Angelman syndrome 1 [32]. One gene showed no homology to known genes. Thus, these five genes, together with the two peroxidase genes, are 
potential positional candidates for Rphq2. Further refinement of the candidate gene list will require knowledge of the role of these genes in defence response and correlation of transcript levels with resistance/susceptibility. Many more genes (i.e. 31) were identified as being differentially expressed and located at the $R p h q 3$ region. This is expected given the larger interval of the QTL (28 cM for Rphq3 vs. $4 \mathrm{cM}$ for Rphq2) and Rphq3 may, therefore, account for differential expression of most of the genes with unassigned map positions. Functionally, none of the differentially expressed genes at $R p h q 2$ or $R p h q 3$ appear to be obvious candidates for a regulator of HvERF4.

Many defence genes encoding PR proteins and components of the phenylpropanoid pathway such as phenylalanine ammonia lyase (PAL) were, as would be expected, Ph-responsive. PR genes encoding beta-1,3-glucanases, chitinases and thaumatin-like proteins exhibit strong in vitro anti-fungal activity [33] and numerous studies have shown that transgenic plants expressing PR-proteins have significant improvement of disease resistance [34-37]. PAL, the first committed enzyme in the phenylpropanoid pathway, is involved in synthesis of both phytoalexins and lignin. Phytoalexins are antimicrobial while lignin synthesis contributes to formation of papillae, which are physical barriers against cell wall penetration by the pathogen [38]. As part of the general response to pathogen infection, few of the genes fell into these categories co-located at the two QTL for partial resistance. One exception, unigene25195, encoding a chitinase (PR3), co-located at $R p h q 2$, was $P h$-responsive and differentially expressed between $R p h q 2$ and $R p h q 2$. However, it was not prioritized as a candidate for $R p h q 2$ since the higher level of gene expression was associated with the susceptibility allele Rphq2. Whereas a number of defence genes were activated in response to $\mathrm{Ph}$-infection, none was found to be a promising candidate for Rphq2 or $R p h q 3$. Our results support the notion that components of the general defence response have incremental, rather than deterministic, roles in the outcome of an interaction between a plant and a pathogen [39]. Many attempts to identify genes for disease resistance have highlighted those involved in signal transduction $[40,41]$ or physiological and cellular functions [42,43] rather than defence per se $[44,45]$.

$P h$-infection triggers a broad range of biological responses with defence response genes being significantly over-represented. Of note is a set of genes encoding receptor-like kinase (RLK), receptor-like proteins (RLP), WRKY, MAPK and PR proteins (Additional File 1, Table S1), which form a complete and well-explored defence signalling cascade starting with the perception of PAMPs, activation of WRKY transcription factors and the subsequent induction of PR proteins [3,46]. Our results also suggest that, in the absence of cognate
$R$ genes to $P$. hordei, plants still mount reactions similar to $R$-gene mediated responses as indicated by the significant up-regulation of genes coding for $R$ gene (-like) proteins and marker genes for oxidative burst such glutathione S-transferase and peroxidase. Although no obvious $R$ genes were identified as candidates for the QTL in this study, $R$ gene-like mediated responses may contribute to basal resistance as a complementary mechanism to PAMP-triggered defence responses. Support for this is provided by observations that resistance QTL are often coincident with the location of $R$-gene homologues [47-51] and that mutated $R$ genes can induce a resistance phenotype similar to quantitative resistance controlled by multiple genes [52-54].

One striking characteristic of the responses to $\mathrm{Ph}$ infection was the activation of signalling pathways related to a broad range of plant hormones including ethylene, gibberellins, auxin, and brassinosteroid as indicated by the up-regulation of genes encoding ethylene-responsive transcription factors, ACC oxidase, auxin-responsive proteins, brassinosteroid insensitive 1-associated receptor kinase 1 (BAK1), gibberellin receptors and a DELLA protein (Additional File 1, Table S1). All of these hormones have been reported to be involved in plant defence responses [55-57] and various defence pathways are interconnected through hormonemediated signalling pathways forming complex regulatory networks $[55,56,58-60]$. Here, the identification of the ethylene-responsive factor $H v E R F 4$ as a putative link between pathogen perception and response is consistent with a role for differential hormone signalling in partial resistance. Understanding the role of $R p h q 2$ of $R p h q 3$ in initiating and coordinating the response requires further work.

Substantial overlap of $\mathrm{Ph}$-responsive genes was identified in super-susceptible (L94) and partially-resistant (Vada) lines. Over $70 \%$ of $\mathrm{Ph}$-responsive genes were detected in both L94 and Vada and had the same expression patterns (up- or down-regulation) in both lines. An even higher percentage of overlapping $\mathrm{Ph}$ responsive genes (79\%) was discovered in both Steptoe and Morex, two cultivars with similar and intermediate level of partial resistance. Given that these lines are genetically diverse, we conclude that barley lines without known cognate $R$ genes to $P$. hordei exhibit similar responses at the transcriptional level, and that observed differences are largely quantitative. Similar findings have been observed in the comparison between compatible and incompatible interactions [61-63]. A small proportion (7\%) of $\mathrm{Ph}$-responsive genes in this study did appear to be resistant/susceptible line-specific and it may be that they determine part of the observed phenotypic differences between lines. However, in $P h$-infected leaves we found no evidence for their differential 
expression in the comparisons between the two QTLNILs and their respective recurrent parents. Therefore, if the variation in resistance, accounted for by $R p h q 2$ or $R p h q 3$, is regulated at the transcriptional level, these are not strong candidate genes.

We generated a robust expression data set in reciprocal Rphq2/Rphq3 QTL-NILs at $18 \mathrm{hpi}$, which is the timepoint previously described as being the most critical during $P$. hordei invasion in barley [25]. However, we realise that transcriptional re-programming in response to pathogen infection is a dynamic and complex process and that defence-associated genes respond to input stimuli with different timing and amplitude. A limitation of our experiment is, therefore, that defence response scenarios constructed on the transcriptional profiles of the $802 \mathrm{Ph}$-responsive genes identified here is simply a snapshot of a dynamic process, at the point when infection hyphae have just attempted penetration of the host cells forming haustoria [25]. To extend our understanding of the complex regulatory mechanisms occurring during defence against $P$. hordei, a more comprehensive investigation would involve sampling at multiple timepoints covering the whole infection period.

\section{Conclusions}

Differential expression with QTL-NILs identifies genes predominantly located at the target region(s) providing both transcriptional and positional candidate genes underlying the QTL. Positional analysis of the differentially expressed genes relative to the QTL has the potential to discover regulatory relays initiated from genes within the QTL.

\section{Methods}

\section{Plant materials}

The plant materials used in this study included both recurrent parental lines L94 (highly susceptible to P. hordei) and Vada (high level of partial resistance to $P$. hordei) and the QTL-NIL named L94-Rphq2+3 and Vada-Rphq2+3 according to the introgressed resistance/ susceptibility QTL alleles. Gene symbol ' $R p h q$ ' refers to the resistance allele of the QTL, i.e. the allele contributed by Vada, and ' $r p h q$ ' refers to the susceptibility L94 allele. Neither of these cultivars carries a cognate $R$-gene to $P$. hordei. The NIL 'L94-Rphq2+3' was previously developed through a marker-assisted backcross programme by incorporating leaf rust resistance alleles $R p h q 2$ and Rphq3 from Vada into L94 susceptible genetic background, whereas the NIL Vada-rphq2+3 was generated by reciprocally incorporating the corresponding susceptibility QTL alleles rphq2 and $r p h q 3$ from L94 into Vada genetic background [18]. The resulting resistance levels (relative latency period in hours) of the NILs are $120 \pm 1.77$ for L94-Rphq2+3, $106 \pm 2.54$ for
Vada-Rphq2+3, as compared to $100 \pm 1.77$ for L94 and $127 \pm 1.80$ for Vada [18]. The genetic lengths of the two introgression segments on chromosome $2 \mathrm{H}$ were 4.6 cM for Rphq2 and 4.4 cM for Rphq2; the two QTL segments on chromosome $6 \mathrm{H}$ were $22.6 \mathrm{cM}$ for $R p h q 3$ and $45.8 \mathrm{cM}$ for rphq3 [18].

Plant growth and leaf inoculations were performed as previously described [25]. The parental lines L94 and Vada and their QTL-NILs, each with 10 seedlings were grown in one tray $(37 \times 39 \mathrm{~cm})$ in two rows $30 \mathrm{~cm}$ apart. A total of eight trays were prepared, with four each used as biological replicates for pathogen inoculation and mock inoculation. The plant growth conditions were as described by Chen et al. [25].

\section{Pathogen inoculation}

Inoculation with $P$. hordei isolate 1.2 .1 was performed on 9-day old seedlings when the first leaf was fully developed and the second leaf was emerging. Leaves were laid horizontal and gently fixed over the soil prior to inoculation. The inoculation was described in Chen et al. [25]. Per plant tray, $8 \mathrm{mg}$ of urediospores plus 32 $\mathrm{mg}$ of Lycopodium spores (added as a carrier) were thoroughly mixed by vortexing and applied to the adaxial sides of the seedling leaves using a settling tower inoculation facility. This amount of spores corresponds to a deposition of about 500 spores per $\mathrm{cm}^{2}$. Mock inoculation of parental lines was carried out using $40 \mathrm{mg}$ of Lycopodium spores only. All trays were transferred to a dark dew chamber at $18^{\circ} \mathrm{C}$ and $100 \%$ relative humidity for 10 hours overnight, before being placed in the glasshouse for infection development.

\section{Leaf sampling}

At $18 \mathrm{hpi}$, both pathogen- and mock-inoculated leaf blades of each replicate and treatment were collected separately into falcon tubes and immediately flashfrozen in liquid nitrogen before being stored at $-80^{\circ} \mathrm{C}$ until use.

\section{RNA isolation, labelling and microarray platform}

RNA isolation was done using the $\mathrm{TRIZOL}^{\circ}$ reagent according to the manufacturer's protocol. cDNA synthesis, labeling and hybridization were performed following the optimized protocol developed by the Sequencing \& Microarray Facility at SCRI. The Agilent $8 \times 15 \mathrm{k}$ format custom array system was used as the platform for RNA profiling. Detailed protocols are described in Chen et al. [25].

\section{Sample layout on the $8 \times 15 \mathrm{k}$ Agilent arrays}

The Agilent platform may be used as a two-colour microarray system allowing two differentially-labeled samples to be tested on a single array. We used three 
Table 2 Microarray experimental design

\begin{tabular}{|c|c|c|c|c|c|c|c|}
\hline \multirow[t]{2}{*}{ Array slide } & \multirow[t]{2}{*}{ Replicate } & \multicolumn{6}{|c|}{ Sample pairs } \\
\hline & & Name & Treatment & Label & Name & Treatment & Label \\
\hline 1 & I & L94 & Mock & C3 & L94 & Ph-infected & C5 \\
\hline 1 & I & Vada & Mock & C3 & Vada & Ph-infected & C5 \\
\hline 1 & $\|$ & L94 & Mock & C3 & L94 & Ph-infected & C5 \\
\hline 1 & $\|$ & Vada & Mock & C3 & Vada & Ph-infected & C5 \\
\hline 1 & III & L94 & Mock & $\mathrm{C} 5$ & L94 & Ph-infected & $\mathrm{C} 3$ \\
\hline 1 & III & Vada & Mock & C5 & Vada & Ph-infected & C3 \\
\hline 1 & IV & L94 & Mock & C5 & L94 & Ph-infected & C3 \\
\hline 1 & IV & Vada & Mock & C5 & Vada & Ph-infected & C3 \\
\hline 2 & 1 & L94 & Ph-infected & C3 & Vada & Ph-infected & C5 \\
\hline 2 & $\|$ & L94 & Ph-infected & C5 & Vada & Ph-infected & $\mathrm{C} 3$ \\
\hline 2 & III & L94 & Ph-infected & C3 & Vada & Ph-infected & C5 \\
\hline 2 & IV & L94 & Ph-infected & $\mathrm{C} 5$ & Vada & Ph-infected & $\mathrm{C} 3$ \\
\hline 3 & 1 & L94 & Ph-infected & $\mathrm{C} 3$ & L94-Rphq2+3 & Ph-infected & $C 5$ \\
\hline 3 & 1 & Vada & Ph-infected & C3 & Vada- Rphq2+3 & Ph-infected & C5 \\
\hline 3 & $\|$ & L94 & Ph-infected & C5 & L94- Rphq2+3 & Ph-infected & $\mathrm{C} 3$ \\
\hline 3 & $\|$ & Vada & Ph-infected & C5 & Vada- Rphq2+3 & Ph-infected & C3 \\
\hline 3 & III & L94 & Ph-infected & C3 & L94- Rphq2+3 & Ph-infected & C5 \\
\hline 3 & III & Vada & Ph-infected & C3 & Vada- Rphq2+3 & Ph-infected & C5 \\
\hline 3 & IV & L94 & Ph-infected & C5 & L94- Rphq2+3 & Ph-infected & C3 \\
\hline 3 & IV & Vada & Ph-infected & $\mathrm{C} 5$ & Vada- Rphq2+3 & Ph-infected & C3 \\
\hline
\end{tabular}

Array slide 1: Ph-infected vs. mock-inoculated controls for Ph-responsive genes; Array slide 2: Ph-infected L94 vs. Ph-infected Vada for differentially expressed genes; Array slide 3: Ph-infected parents vs. Ph-infected QTL-NILs for QTL specific and differentially expressed genes.

different sample layouts depending upon the biological questions to be addressed: 1) RNA samples from $P h$ infected parents and mock-inoculated controls (four replicates) were hybridized onto single arrays to identify Ph-responsive genes (array slide 1 in Table 2); 2) RNA samples from $\mathrm{Ph}$-infected L94 and Vada (four replicates) were hybridized onto single arrays to test genome-wide differential expression (slide 2 in Table 2); 3) RNA samples from Ph-infected L94 and L94-Rphq2+3 or Vada and Vada-Rphq $2+3$ were put on single arrays with four replicates ( 8 arrays) (slide 3 in Table 2 ) to compare expression levels of parental lines with their respective NILs. In all sample layouts, a balanced dye swap strategy was applied as indicated in the Table 2.

\section{Deposition of microarray data}

The raw microarray data and relevant experimental metadata, which are MIAME (Minimum Information About a Microarray Experiment) compliant, are deposited at the ArrayExpress microarray data archive http:// www.ebi.ac.uk/microarray-as/ae/ at the European Bioinformatics Institute (accession numbers: E-TABM-980).

Data extraction, normalisation and significance criteria for differential expression

Data extraction and normalisation were done independently for the three different experiments with
GeneSpring (v.7.3) software as described previously [25]. Briefly, dye swap was corrected in relevant samples, followed by Lowess (LOcally WEighted polynomial regreSSion) normalisation to minimize differences in dye incorporation efficiency in a two-channel microarray platform [64]. Differentially expressed genes were first selected on fold change $>2$ followed by a Students $t$-test on log-transformed normalised ratio data, setting the False Discovery Rate (FDR) to 0.05 .

\section{Additional material}

Additional file 1: Table S1. Expression information of Ph-reponsive genes identified on L94 and Vada (Ph-infected vs. mock control).

Additional file 2: Table S2. Expression information of resistant/ susceptible line-specific and Ph-responsive genes.

Additional file 3: Table S3. Expression of the resistant/susceptible linespecific genes (upper/lower panel) reproduced as Ph-responsive genes in St and Mx.

Additional file 4: Table S4. Genome-wide differentially expressed genes in Ph-infected seedlings between Vada and L94.

Additional file 5: Table S5. List of the 55 differentially expressed genes showing expression ratios and $p$-values in different comparisons and map position of eQTL and corresponding genes from different sources.

\section{Abbreviations}

Ph: Puccinia hordei; QTL: quantitative trait loci; eQTL: expression QTL; QTL-NIL: QTL-specific nearly isogenic line; RIL: recombinant inbred line, PAMP: 
pathogen-associated molecular pattern; PTI: PAMP-triggered immunity; ETI: effector-triggered immunity, FDR: false discovery rate; GO: gene ontology; LOD: log of odds; LRS: likelihood ratio statistics; FC: fold change; SFP: single feature polymorphism; TDM: transcript derived marker.

\section{Acknowledgements}

We gratefully acknowledge J McNicol, C Hackett and D Roberts for valuable discussions concerning the experimental and custom array design; F Yeo, A Gonzalez, Z Kohutova, F Meijer-Dekens, R Aghnoum, M Macaulay and K McLean for their kind help with sampling; and Drs A Newton and G Bryan for their critical review of the manuscript. Funding for this experiment was provided by the European Union Bioexploit Grant No. 513959 (FOOD) to RW and RN http://www.bioexploit.net and by Scottish Government Rural and Environment Research and Analysis Directorate (RERAD) Programme 1, Work Package 1 http://www.programme1.net/programmes. We thank Dr R Wise for access to unpublished eQTL data uploaded in the GeneNetwork.

\section{Author details}

${ }^{1}$ Genetics Programme, Scottish Crop Research Institute, Dundee, UK. ${ }^{2}$ Laboratory of Plant Breeding, Graduate School for Experimental Plant Sciences, Wageningen University, Wageningen, The Netherlands. ${ }^{3}$ INRAAgroParisTech, UMR 1290 BIOGER-CPP, Avenue Lucien Brétignières BP01, 78850 Thiverval-Grignon, France.

\section{Authors' contributions}

Conceived and designed the experiments: XC, REN, AD and RW; performed the experiments: $X C_{;}$wrote the paper: $X C$ and RW. Pathogen infection and sampling: REN, XC, TCM and AV. Microarray and data deposition: PH and JM. All authors read and approved the final manuscript.

Received: 4 June 2010 Accepted: 11 November 2010

Published: 11 November 2010

\section{References}

1. Ausubel FM: Are innate immune signaling pathways in plants and animals conserved? Nature Immunology 2005, 6:973-79.

2. Nicaise V, Roux M, Zipfel C: Recent advances in PAMP-triggered immunity against bacteria: pattern recognition receptors watch over and raise the alarm. Plant Physiology 2009, 150:1638-1647.

3. Bent AF, Mackey D: Elicitors, effectors, and $R$ genes: the new paradigm and a lifetime supply of questions. Annual Review of Phytopathology 2007, 45:399-436.

4. Bittel P, Robatzek S: Microbe-associated molecular patterns (MAMPs) probe plant immunity. Current Opinion in Plant Biology 2007, 10:335-341.

5. Niks RE, Marcel TC: Nonhost and basal resistance: how to explain specificity? New Phytologist 2009, 182:817-828.

6. Niks RE: Failure of haustorial development as a factor in slow growth and development of Puccinia hordei in partially resistant barley seedlings. Physiological and Molecular Plant Pathology 1986, 28:309-322.

7. O'Connell RJ, Panstruga R: Tete a tete inside a plant cell: establishing compatibility between plants and biotrophic fungi and oomycetes. New Phytologist 2006, 171:699-718.

8. Heath MC: Cellular interactions between biotrophic fungal pathogens and host or nonhost plants. Canadian Journal of Plant Pathology 2002, 24:259-264.

9. Collins NC, Niks RE, Schulze-Lefert P: Resistance to cereal rusts at the plant cell wall - what can we learn from other host-pathogen systems? Australian Journal of Agricultural Research 2007, 58:476-489.

10. Hardham AR, Jones DA, Takemoto D: Cytoskeleton and cell wall function in penetration resistance. Current Opinion in Plant Biology 2007, 10:342-348.

11. Qi X, Niks RE, Stam P, Lindhout P: Identification of QTLs for partial resistance to leaf rust (Puccinia hordei) in barley. Theoretical and Applied Genetics 1998, 96:1205-1215.

12. Qi X, Jiang G, Chen W, Niks RE, Stam P, Lindhout P: Isolate-specific QTLs for partial resistance to Puccinia hordei in barley. Theoretical and Applied Genetics 1999, 99:877-884.

13. Jafary H, Szabo L, Niks RE: Innate nonhost immunity in barley to different heterologous rust fungi is controlled by sets of resistance genes with overlapping specificities. Molecular Plant-Microbe Interaction 2006, 19:1270-1279.
14. Jafary H, Albertazzi G, Marcel TC, Niks RE: High diversity of genes for nonhost resistance of barley to heterologous rust fungi. Genetics 2008, 178:2327-2339.

15. Marcel TC, Varshney RK, Barbieri M, Jafary H, de Kock MJD, Graner A, Niks RE: A high-density consensus map of barley to compare the distribution of QTLs for partial resistance to Puccinia hordei and of defence gene homologues. Theoretical and Applied Genetics 2007, 114:487-500.

16. Marcel TC, B Gorguet M, Truong Ta, Kohutova Z, Vels A, Niks RE: Isolatespecificity of quantitative trait loci for partial resistance of barley to Puccinia hordei confirmed in mapping populations and near-isogenic lines. New Phytologist 2008, 177:743-755.

17. van Berloo R, Aalbers H, Werkman A, Niks RE: Resistance QTL confirmed through development of QTL-NILs for barley leaf rust resistance. Molecular Breeding 2001, 8:187-195.

18. Marcel TC, Aghnoum R, Durand J, Varshney RK, Niks RE: Dissection of the barley $2 \mathrm{~L} 1.0$ region carrying the 'Laevigatum' quantitative resistance gene to leaf rust using near-isogenic lines (NIL) and subNIL. Molecular Plant-Microbe Interactions 2007, 20:1604-1615.

19. Wise RP, Moscou MJ, Bogdanove AJ, Whitham SA: Transcript profiling in host-pathogen interactions. Annual Review of Phytopathology 2007, 45:329-369.

20. Jansen RC, Nap JP: Genetical genomics: the added value from segregation. Trends in Genetics 2001, 17:388-391.

21. Druka A, Potokina E, Luo Z, Jiang N, Chen X, Kearsey M, Waugh R: eQTL analysis in Plants. Plant Biotechnology Journal 2009, 8:10-27.

22. Potokina E, Druka A, Luo Z, Wise R, Waugh R, Kearsey MJ: Gene expression quantitative trait locus analysis of 16000 barley genes reveals a complex pattern of genome-wide transcriptional regulation. The Plant Journal 2008, 53:90-101.

23. Potokina E, Druka A, Luo Z, Moscou M, Wise R, Waugh R, Kearsey MJ: Tissue-dependent limited pleiotropy affects gene expression in barley. The Plant Journal 2008, 56:287-296.

24. Druka A, Potokina E, Luo Z, Bonar N, Druka I, Zhang L, Marshall DF, Kearsey $M$, Waugh R: Exploiting regulatory variation to identify genes underlying quantitative resistance to the wheat stem rust pathogen Puccinia graminis f. sp tritici in barley. Theoretical and Applied Genetics 2008, 117:261-272.

25. Chen X, Hackett CA, Niks RE, Hedley PE, Booth C, Druka A, Marcel TC, Vels A, Bayer M, Milne I, Morris J, Ramsay L, Marshall D, Cardle L, Waugh R: An eQTL analysis of partial resistance to Puccinia hordei in barley. PLoS ONE 2010, 5:e8598.

26. Luo ZW, Potokina E, Druka A, Wise R, Waugh R, Kearsey MJ: SFP genotyping from Affymetrix arrays is robust but largely detects cisacting expression regulators. Genetics 2007, 176:789-800.

27. Glazebrook J: Genes controlling expression of defense responses in Arabidopsis: 2001 status. Current Opinion in Plant Biology 2001, 4:301-308.

28. Close TJ, Bhat PR, Lonardi S, Wu Y, Rostoks N, Ramsay L, Druka A, Stein N, Svensson JT, Wanamaker S, Bozdag S, Roose ML, Moscou MJ, Chao S, Varshney RK, Szúcs P, Sato K, Hayes PM, Matthews DE, Kleinhofs A, Muehlbauer GJ, DeYoung J, Marshall DF, Madishetty K, Fenton RD, Condamine P, Graner A, Waugh R: Development and implementation of high-throughput SNP genotyping in barley. BMC Genomics 2009, 10:582.

29. Hansen BG, Halkier BA, Kliebenstein DJ: Identifying the molecular basis of QTLs: eQTLs add a new dimension. Trends in Plant Science 2008, 13:72-77.

30. Yang Z, Tian L, Latoszek-Green M, Brown D, Wu K: Arabidopsis ERF4 is a transcriptional repressor capable of modulating ethylene and abscisic acid responses. Plant Molecular Biology 2005, 58:585-596.

31. Shinshi H, Usami S, Ohme-Takagi M: Identification of an ethyleneresponsive region in the promoter of tobacco class I chitinase gene. Plant Molecular Biology 1995, 27:923-932.

32. Rainier S, Chai JH, Tokarz D, Nicholls RD, Fink JK: NIPA1 gene mutations cause autosomal dominant hereditary spastic paraplegia (SPG6). American Journal of Human Genetics 2003, 73:967-971.

33. Mauch $F$, Hadwiger $L A$, Boller $T$ : Antifungal hydrolases in pea tissue. 1. Purification and characterization of two chitinases and $\beta-1,3$-glucanase differentially regulated during development and in response to fungal infection. Plant Physiology 1988, 87:325-333.

34. Broglie K, Chet I, Holliday M, Cressman R, Biddle Ph, Knowlton S, Mauvais CJ, Broglie R: Transgenic plants with enhanced resistance to the fungal pathogen Rhizoctonia solani. Science 1991, 254:1194-1197. 
35. Zhu Q, Maher EA, Masoud S, Dixon RA, Lamb C: Enhanced protection against fungal attack by constitutive co-expression of chitinase and glucanase genes in transgenic tobacco. Bio/Technology 1994, 12:807-812.

36. Lin W, Anuratha CS, Datta K, Potrykus I, Muthukrishnan S, Datta SK: Genetic engineering of rice for resistance to sheath blight. Bio/Technology 1995, 13:686-691.

37. Datta K, Velazhahan R, Oliva N, Ona I, Mew T, Khush GS, Muthukrishnan S, Datta SK: Over-expression of the cloned rice thaumatin-like protein (PR5) gene in transgenic rice plants enhances environmental friendly resistance to Rhizoctonia solani causing sheath blight disease. Theoretical and Applied Genetics 1999, 98:1138-1145.

38. Dixon RA: Natural products and plant disease resistance. Nature 2001, 411:843-847.

39. Collinge DB, Jensen MK, Lyngkjaer MF, Rung J: How can we exploit functional genomics approaches for understanding the nature of plant defences? Barley as a case study. European Journal of Plant Pathology 2008, 121:257-266.

40. Glazebrook J: Contrasting mechanisms of defense against biotrophic and necrotrophic pathogens. Annual Review of Phytopathology 2005, 43:205-227.

41. Panstruga $R$, Schulze-Lefer P: Live and let live: insights into powdery mildew disease and resistance. Molecular Plant Pathology 2002, 3:495-502.

42. Krattinger SG, Lagudah ES, Spielmeyer W, Singh RP, Huerta-Espino J, McFadden $H$, Bossolini E, Selter LL, Keller B: A putative ABC transporter confers durable resistance to multiple fungal pathogens in wheat. Science 2009, 323:1360-1363.

43. Fu D, Uauy C, Distelfeld A, Blechl A, Epstein L, Chen X, Sela H, Fahima T, Dubcovsky J: A kinase-START gene confers temperature-dependent resistance to wheat stripe rust. Science 2009, 323:1357-1360.

44. Field B, Jordan F, Osbourn A: First encounters-Deployment of defencerelated natural products by plants. New Phytologist 2006, 172:193-207.

45. van Loon LC, Rep M, Pieterse CMJ: Significance of inducible defenserelated proteins in infected plants. Annual Review of Phytopathology 2006, 44:135-162.

46. Bittel P, Robatzek S: Microbe-associated molecular patterns (MAMPs) probe plant immunity. Current Opinion in Plant Biology 2007, 10:335-341.

47. Wagner C, Schweizer G, Kraemer M, Dehmer-Badani AG, Ordon F, Friedt W: The complex quantitative barley-Rhynchosporium secalis interaction: newly identified QTL may represent already known resistance genes. Theoretical and Applied Genetics 2008, 118:113-122.

48. Tan MYA, Hutten RCB, Celis C, Park TH, Niks RE, Visser RGF, van Eck HJ: The RPi-mcd1 locus from Solanum microdontum involved in resistance to Phytophthora infestans, causing a delay in infection, maps on potato chromosome 4 in a cluster of NBS-LRR genes. Molecular Plant-Microbe Interactions 2008, 21:909-918.

49. Xiao W, Zhao J, Fan S, Li L, Dai J, Xu M: Mapping of genome-wide resistance gene analogs (RGAs) in maize (Zea mays L.). Theoretical and Applied Genetics 2007, 115:501-508.

50. Zimnoch-Guzowska E, Marczewski W, Lebecka R, Flis B, Schäfer-Pregl R, Salaminin F, Gebhardt C: QTL analysis of new sources of resistance to Erwinia carotovora ssp. atroseptica in potato done by AFLP, RFLP, and resistance-gene-like markers. Crop Science 2000, 40:1156-1167.

51. Pflieger S, Lefebvre V, Caranta C, Blattes A, Goffinet B, Palloix A: Disease resistance gene analogs as candidates for QTLs involved in pepperpathogen interactions. Genome 1999, 42:1100-1110.

52. Wang GL, Ruan DL, Song WY, Sideris S, Chen L, Pi LY, Zhang S, Zhang Z, Fauquet C, Gaut BS, Whalen MC, Ronald P: Xa21D encodes a receptor-like molecule with a leucine-rich repeat that determines race-specific recognition and is subject to adaptative evolution. The Plant Cell 1998, 10:765-779.

53. Anderson PA, Lawrence GJ, Morrish BC, Ayliffe MA, Finnegan EJ, Ellis JG: Inactivation of the flax rust resistance gene $M$ associated with loss of a repeated unit within the leucine-rich repeat coding region. The Plant Cell 1997, 9:641-651.

54. Ori N, Eshed Y, Paran I, Presting G, Aviv D, Tanksley S, Zamir D, Fluhr R: The I2C family from the wilt disease resistance locus $I 2$ belongs to the nucleotide binding, leucine-rich repeat superfamily of plant resistance genes. The Plant Cell 1997, 9:521-532.

55. Bari $R$, Jones JD: Role of plant hormones in plant defence responses. Plant Molecular Biology 2009, 69:473-88.
56. Kazan K, Manners JM: Linking development to defense: auxin in plantpathogen interactions. Trends in Plant Science 2009, 14:373-382.

57. Shan L, He P, Li J, Heese A, Peck SC, Nurnberger T, Martin GB, Sheen J: Bacterial effectors target the common signaling partner BAK1 to disrupt multiple MAMP receptor-signaling complexes and impede plant immunity. Cell Host \& Microbe 2008, 4:17-27.

58. Krishna P: Brassinosteroid-mediated stress responses. Journal of Plant Growth Regulation 2003, 22:289-297.

59. Yi HC, Joo S, Nam KH, Lee JS, Kang BG, Kim WT: Auxin and brassinosteroid differentially regulate the expression of three members of the 1aminocyclopropane-1-carboxylate synthase gene family in mung bean (Vigna radiata L.). Plant Molecular Biology 1999, 41:443-454.

60. Muessig C, Lisso J, Coll-Garcia D, Altmann T: Molecular analysis of brassinosteroid action. Plant Biology 2006, 8:291-296.

61. Eulgem T: Regulation of the Arabidopsis defense transcriptome. Trends in Plant Science 2005, 10:71-78.

62. Maleck K, Levine A, Eulgem T, Morgan A, Schmid J, Lawton KA, Dangl JL, Dietrich RA: The transcriptome of Arabidopsis thaliana during systemic acquired resistance. Nature Genetics 2000, 26:403-410.

63. Caldo RA, Nettleton D, Wise RP: Interaction-dependent gene expression in Mla-specified response to barley powdery mildew. The Plant Cell 2004, 16:2514-2528.

64. Yang YH, Dudoit S, Luu P, Lin DM, Peng V, Ngai J, Speed TP: Normalization for CDNA microarray data: a robust composite method addressing single and multiple slide systematic variation. Nucleic Acids Research 2002, 30:4e15.

doi:10.1186/1471-2164-11-629

Cite this article as: Chen et al: Differential gene expression in nearly isogenic lines with QTL for partial resistance to Puccinia hordei in barley. BMC Genomics 2010 11:629.

\section{Submit your next manuscript to BioMed Central and take full advantage of:}

- Convenient online submission

- Thorough peer review

- No space constraints or color figure charges

- Immediate publication on acceptance

- Inclusion in PubMed, CAS, Scopus and Google Scholar

- Research which is freely available for redistribution

Submit your manuscript at www.biomedcentral.com/submit
C Biomed Central 